\title{
Obstruction to the Existence of Metric whose Curvature has Umbilical Hessian in a K-Surface XiUXiong Chen
}

\section{Introduction.}

1.1. Introduction to the problem. Let $M$ be any compact, oriented smooth Riemannian surface without a boundary, and $M_{\left\{\alpha_{1}, \alpha_{2}, \ldots, \alpha_{n}\right\}}$ (where $\alpha_{i}>0, \forall 1 \leq i \leq n$ ) denote a $K$-surface associated with $M$. A Riemannian metric $g$ is said to be well defined or smooth in $M_{\left\{\alpha_{1}, \alpha_{2}, \ldots, \alpha_{n}\right\}}$ if it satisfies the following two conditions: (1) $g$ is smooth everywhere on $M$ except in a set of singular points $\left\{p_{1}, p_{2}, \cdots, p_{n}\right\}$. (2) For any $i(1 \leq i \leq n)$, the metric $g$ has a singular angle of $2 \pi \alpha_{i}$ at the point $p_{i}$. Therefore, the scalar curvture of any metric which is defined in a K-surface must have a $\delta$-distribution in $M$; and have a concentrated weight at every singular point of the metric. (This is the reason why we called it K-surface.) Two smooth Riemannian metrics on $M_{\left\{\alpha_{1}, \alpha_{2}, \ldots, \alpha_{n}\right\}}$ are pointwise conformal to each other if they are related by a multiple of a smooth positive function on $M$. An old and enduring problem in 2 dimensional Riemannian geometry and real analysis is whether there always exists a "best metric" in any pointwise conformal class in a K-surface. Of course, the answer to this question really depends on what the "best metric" is. In this paper, we propose to consider two metrics as the likely candidates of the "best metric." The first is the extremal metric, which is a critical point of an energy functional ( $L^{2}$ norm of the scalar curvature function). The second metric has a property that its scalar curvature function has an umbilical Hessian. In other words, the 2nd covariant derivative tensor of the scalar curvature function of the metric is pointwise proportional to the metric tensor. For convenience, we use the abbreviation "HCMU" to denote this second metric (the Hessian of the Curvature of the Metric is Umbilical). Both extremal metrics and HCMU metrics are defined in section 2. The extremal metric includes the HCMU metric as a special case; both metrics include the constant curvature metric as a special case. While we believe that the extremal metric is the ultimate 
choice of the "best metric," we concentrate on studying to what extent the topology of the underlying surface is determined by the presence of a HCMU metric in this paper. Our main result is:

Theorem 1. Let $g$ be a HCMU metric in a K-surface $M_{\left\{\alpha_{1}, \alpha_{2}, \cdots, \alpha_{n}\right\}}$. Then the Euler-Lagrange character of the underlying surface should be determined by

$$
\chi(M)=\sum_{i=1}^{j}\left(1-\alpha_{i}\right)+(n-j)+s
$$

where $s$ is the mumber of critical points of the curvaure $K_{g}$ (excluding the singular points of $g)$. Here we assume that $\alpha_{1}, \alpha_{2}, \ldots . \alpha_{k}(0 \leq k \leq n)$ are the only integers in the set of prescribed angles $\left\{\alpha_{1}, \alpha_{2}, \cdots, \alpha_{n}\right\}$; and assume that $\left\{p_{j+1}, \cdots, p_{k}\right\}$ are the only local extremal points of $K_{g}$ in the set of singular points $\left\{p_{j}, 0 \leq j \leq k\right\}$.

This theorem has an important implication:

Theorem 2. If none of the prescribed angles in a $K$-surface is an integer multiple of $2 \pi$, this K-surface supports a HCMU metric if and only if it is a football with two different angles at the two poles. Furthermore, any HCMU metric in these footballs must be rotationally symmetric and uniquely determined by the area.

Another corollay of Theorem 1 is:

Corollary 1. If $g$ is a HCMU metric in a K-surface $M_{\alpha_{1}, \alpha_{2}, \cdots, \alpha_{n}}$, then

1. The curvature $K_{g}$ is continuous on all of the singular points of $g$.

2. Any singular point of metric $g$ whose singular angle is not an integer multiple of $2 \pi$ (i.e., $\left\{p_{k+1}, p_{k+2}, \cdots, p_{n}\right\}$ ), must be a local extremal point of $K_{g}$.

3. The curvature $K_{g}$ does not have any smooth saddle point.

4. $\sum_{i=1}^{k}\left(1-\alpha_{i}\right)+(n-k) \leq \chi(M)$. 
Theorem 1 was proved by studying the structure of a special Killing vector field. The method is elementary, geometrical. The most important step is to show that this Killing vector field must only have a finite number of singular points. In the proof, it is not assumed that the scalar curvature of HCMU metric is bounded. However, we do assume any HCMU metric must have a finite energy and area since it is a critical point of the energy functional.

1.2 Historical Remarks. The classical uniformization theorem asserts that every pointwise conformal class, in a smooth surface without a boundary, admits a constant curvature metric. Unfortunately, the parallel statement in a K-surface is not always true. For instance, there is no constant curvature metric in a football if the two angles at the two poles are different. However, every football does support a HCMU metric. Since a constant curvature metric is also a HCMU metric, one might be tempted to ask if there always exists a HCMU metric in any pointwise conformal class in a $\mathrm{K}$ surface. The answer to this question is no; and we will give some necessary conditions for the existence of a HCMU metric in a K-surface. The remaining question is: what should the "best metric" be so that every pointwise conformal class will admit one? To answer this question, we propose the following problem:

Conjecture/Problem 1. Is any smooth Riemannian metric in a $\mathrm{K}$ surface pointwise conformal to an extremal metric?

Observe that a HCMU metric is also an extremal metric; and a constant scalar curvature metric is also a HCMU metric. According to [10], these three kinds of metrics are identical in any smooth surface without a boundary. If the problem 1 had a positive answer, it then included the classical uniformization theorem on a smooth surface as a special case. There have been numerous attempts to generalize the classical uniformization theorem, mainly focusing on the solvability of the scalar curvature equation (2.4) on a given K-surface. Independently, [12] and [13] have found some sufficient conditions for a $\mathrm{K}$-surfaces to admit constant curvature metric. Under some restrictive conditions, [6] gave some necessary conditions for a $\mathrm{K}$-surface to support a constant curvature metric. However, [3] had constructed a sequence of constant curvature metric in $S^{2}$ with 3 singular points, where the singular angles could be made arbitrarily large. Also in [3] , E. Calabi gave a sufficient condition which should be satisfied by the three singular angles, so that $S^{2}$ with these angles will support a constant (positive) curvature 
metric.

There will be no uniformization theroem in a K-surface if we consider only the constant curvature metric as the "best metric." Therefore, we think the extremal metric may be a better candidate for the "best metric." If the problem 1 had been positively solved, it would be clear one should focus on the understanding of the topological obstructions to reduce the equation (2.3) to (2.4) and to reduce the equation (2.1) to (2.3)(these three equations are given in section 2). The present paper will answer to what extent the existence of HCMU metric in a K-surface restricts the topology of the underlying K-surface. Even though a constant curvature metric is also a HCMU metric, they shall be excluded from the discussions here. Therefore, a HCMU metric, in this work, must have a non-constant scalar curvature function.

E. Calabi[4] has constructed a smooth HCMU metric $g^{0}$ in $S_{\{2,2,2\}}^{2}$. It was not known to him whether there exists any other HCMU metric on this $\mathrm{K}$-surface. In [2], E. Calabi shows that any extremal Kähler metric must have maximized symmetry determined by the complex structure. He raised the question if the space of extremal Kähler metrics in same DeRham class is connected and if it could have different energy levels, since any extremal Kähler metric must be a local minimizer. At least in dimension 2, the answer to these questions are all negative. Using the main theorem, another HCMU metrics in $S_{2,2,2}^{2}$ are constructed explicitely. This metric demonstrated less symmetry and is not locally isometric to the metric constructed by E. Calabi; and the two metrics have different energy levels. However, these two metrics are connected in the same DeRham class.

Recently, we learned an ambitious program of Donaldson [18] where he puts a Riemannian metric ${ }^{1}$ in the space of Kähler metrics such that the later becomes an infinite dimensional symmetric space. The conjecture (or question) he raised is whether such a symmetric space is always geodesically convex. His program shows that a positive answer to this conjecture (or question) leads to the uniqueness of the smooth extremal Kähler metric in each Kähler class up to holomorphic transformation. Therefore, the above mentioned non-uniqueness example of degenerated extremal Kähler metrics becomes rather interesting and need to be further understood. Noticed that any HCMU metric is a degenerated extremal Kähler metric. Follow Donaldson's program, in [17], we proved the following results: a) the space of Kähler metrics is convex by $C^{1,1}$ geodesic; b) it is a metric space; c)the extremal Kähler metric is unique in each Kähler class if the first Chern class

\footnotetext{
${ }^{1}$ this metric is also introduced by Mabuchi [20] and S. Semmes in [19].
} 
is strictly negative. The details of these results will be appeared else where. These results certainly make these examples we constructed here much more interesting.

Another significant implication of these examples is that the solution of the Euler-Lagrange equation 2.1 is not unique, even under a very strigent boundary conditions. Both Calabi's metric and the one constructed by author have uniformly bounded curvature; the two metrics differ in the neighborhood of the singular points only by a smooth positive function on $S^{2}$. Calabi's metric has more symmetry and has less energy. We conjecture it is a real minimizer in its DeRham class.

A few words about the organization of this paper. In section 2, we introduce the vaiational problem, give two versions of Euler-Lagrange equations and then prove the two versions are equivalent. Also in this section, we define the three kinds of metrics discussed here. In section 3 , we first show Calabi's construction of a HCMU metric in $S_{2,2,2}$. Then we use the Theorem 1 to analyze all the HCMU metrics in $S_{2,2,2}^{2}$. Using these information, we construct explicitely two different HCMU metrics in $S_{2,2,2}^{2}$. In section 4 , we study the Killing vector field of a HCMU metric. In section 5 , we give the proof of the main theorems. In section 6 , we give proof to a technical lemma which gives an estimate of the curvature function near a conical singular point.

Acknowledgments. The author wishes to thank Professor M. C. Wang for many helpful and stimulating conversations during the course of this work. Thank also to Professor R. Schoen for his continued support and encouragements. Also to my advisor Professor E. Calabi for his warm encouragements during the past two years.

\section{Euler-Lagrange Equations.}

An extremal metric is a critical point of the following energy functional:

$$
E(g)=\int_{M_{\backslash}\left\{p_{1}, p_{2}, \cdots, p_{n}\right\}} K_{g}^{2} d g .
$$

The appropriate variational space for this functional is the space of all smooth Riemannian metrics with a fixed area in a fixed pointwise conformal class. Let $g_{0}$ be a generic smooth metric in $M_{\alpha_{1}, \alpha_{2}, \cdots, \alpha_{n}}$, the function space is then:

$$
\mathcal{S}\left(g_{0}\right)=\left\{\varphi \in H^{2,2}(M) \mid \int_{M \backslash\left\{p_{1}, p_{2}, \cdots, p_{n}\right\}} e^{2 \varphi} d g_{0}=\int_{M \backslash\left\{p_{1}, p_{2}, \cdots, p_{n}\right\}} d g_{0}\right\}
$$


For any $\varphi \in \mathcal{S}\left(g_{0}\right)$, the corresponding metric $g=e^{2 \varphi} \cdot g_{0}$ is smooth in the K-surface $M_{\alpha_{1}, \alpha_{2}, \cdots, \alpha_{n}}$. Conversely, for any smooth Riemannian metric in $M_{\alpha_{1}, \alpha_{2}, \cdots, \alpha_{n}}$, there exists a function $\varphi \in \mathcal{S}\left(g_{0}\right) \subset H^{2,2}(M)$ such that $g=e^{2 \varphi} \cdot g_{0}$. Observe that the function space is a subset of $H^{2,2}(M)$, not a subset of $H_{l o c}^{2,2}\left(M \backslash\left\{p_{1}, p_{2}, \cdots, p_{n}\right\}\right)$. If a metric is deformed in a pointwise conformal class, the structure of the conical angle at each singular point should be preserved. The Euler-Lagrange equation of this functional is:

$$
\triangle_{g} K_{g}+K_{g}^{2}=C \text { (generic constant). }
$$

Equivalently, in a local complex coordinate chart,

$$
\frac{\partial}{\partial \bar{z}} K_{g, z z}=0
$$

where $K_{g, z z}$ is the 2nd-order covariant derivative of $K_{g}$. We will show these two Euler-Lagrange equations are equivalent shortly. Any metric satisfies the equation (2.1) or (2.2) is called an extremal metric, even if it is only a critical point of the energy functional.

The Euler-Lagrange equation has two special cases. The first special case is:

$$
K_{g_{, z z}}=0 .
$$

Any metric is HCMU if and only if it satisfies this equation (2.3). The second special case is:

$$
K_{g} \equiv c, \quad \text { where } c \text { is a constant. }
$$

For any singular point $p_{i}(1 \leq i \leq n), g$ can be re-written in a local coordinate chart $(\mathcal{U}, z)\left(z\left(p_{i}\right)=0\right)$ as:

$$
g=h(z, \bar{z}) \frac{1}{|z|^{2-2 \alpha}}|d z|^{2}, \quad h(0)>0, \quad \forall z \in \mathcal{U} .
$$

For any $p \notin\left\{p_{1}, p_{2}, \cdots, p_{n}\right\}$, we can choose a local coordinate chart $(\mathcal{U}, z)(z(p)=0)$, such that metric $g$ can be written as:

$$
g=e^{2 \varphi(z, \bar{z})}|d z|^{2}, \quad \forall z \in \mathcal{U} .
$$

The scalar curvature is:

$$
K_{g}=-\frac{\triangle \varphi}{e^{2 \varphi}},
$$


where " $\triangle$ " is the real Laplacian operator with respect to the local Euclidean metric. Equation (2.3) can be re-written as:

$$
K_{g_{, z z}}=\frac{\partial^{2} K_{g}}{\partial z^{2}}-2 \frac{\partial K_{g}}{\partial z} \cdot \frac{\partial \varphi(z, \bar{z})}{\partial z}=0 .
$$

Therefore, the equation for a HCMU metric is not elliptic type. According to equation (2.8), any metric is HCMU if and only if the gradient vector field of its scalar curvature function is holomorphic. This vector field is defined as:

$$
\overrightarrow{\nabla K_{g}}=\sqrt{-1} K_{g}{ }^{, z} \frac{\partial}{\partial z}=\sqrt{-1} e^{-2 \varphi} \cdot \frac{\partial K_{g}}{\partial \bar{z}} \cdot \frac{\partial}{\partial z}
$$

Proposition 1. The "real version" Euler-Lagrange equation (2.1) is equivalent to the "complex version" Euler-Lagrange equation (2.2).

Proof. We first derive the "complex version" Euler-Lagrange equation (2.2) from the "real version" Euler-Lagrange equation (2.1). Observe that the real Laplacian operator and the complex Laplacian operator only differ by a constant. Thus, $\triangle_{g}=2 e^{-2 \varphi} \cdot \frac{\partial}{\partial z} \frac{\partial}{\partial \bar{z}}$. The equation (2.1) can be written as follows:

$$
\frac{2 \frac{\partial}{\partial z} \frac{\partial}{\partial \bar{z}} K_{g}}{e^{2 \varphi}}+R_{g}^{2}=0
$$

By differentiating the above expression with $\frac{\partial}{\partial z}$, the results are:

$$
\frac{2 \frac{\partial}{\partial z} \frac{\partial}{\partial z} \frac{\partial}{\partial \bar{z}} K_{g}}{e^{2 \varphi}}-4 \frac{\frac{\partial \varphi}{\partial z} \frac{\partial}{\partial z} \frac{\partial}{\partial \bar{z}} K_{g}}{e^{2 \varphi}}+2 K_{g} \cdot \frac{\partial}{\partial z} K_{g}=0 .
$$

Exchange the order of differentiation of $K_{g}$ in the first two terms, substitute $K_{g}=-2 \frac{\frac{\partial}{\partial \bar{z}} \frac{\partial}{\partial z} \varphi}{e^{2 \varphi}}$ into the first " $K_{g}$ " of the third term, and then get rid of the common denominator $e^{2 \varphi}$. The above equation then becomes:

$$
2 \frac{\partial}{\partial \bar{z}} \frac{\partial}{\partial z} \frac{\partial}{\partial z} K_{g}-4 \frac{\partial \varphi}{\partial z} \frac{\partial}{\partial \bar{z}} \frac{\partial}{\partial z} K_{g}-4 \frac{\partial}{\partial \bar{z}} \frac{\partial}{\partial z} \varphi \frac{\partial}{\partial z} K_{g}=0 .
$$

The last equation can be rearranged as:

$$
\frac{\partial}{\partial \bar{z}}\left(\frac{\partial}{\partial z} \frac{\partial}{\partial z} K_{g}-2 \frac{\partial}{\partial z} \varphi \frac{\partial}{\partial z} K_{g}\right)=0 .
$$


The last equation is exactly the complex Euler-Lagrange equation(2.2). To derive the real Euler-Lagrange equation(2.1) from the complex equation Euler-Lagrange(2.2), one just go through the above procedures in exactly the reversed order. Therefore, the two equations are equivalent.

Two theorems about the HCMU metric will be given before concluding this section.

Theorem 3. Any HCMU metric must be a local minimizer for the energy functional $E$.

This theorem should be read with care. We consider only those deformations of a metric which vanish in a small neighborhood of the set of prescribed singular points. The proof of this theorem is the same as the proof in [2]. Readers are refered there for detailed proof. Whether a HCMU metric is a local minimizer, with respect to a general deformation which preserves the conical angle structure at each singular point, is not known.

Theorem 4. If the scalar curvature of a HCMU metric is a constant in a small open set, the scalar curvature must be a constant everywhere.

Proof. Let $g$ be a HCMU metric with a scalar curvature function $K_{g}$. The gradient vector field $\overrightarrow{\nabla K_{g}}$ is a holomorphic vector field. If $K_{g}$ is a constant function in a small open set $\mathcal{O} \subset M \backslash\left\{p_{1}, p_{2}, \cdots, p_{n}\right\}$, then $\overrightarrow{\nabla K_{g}}$ vanishes in this small open set. This further implies $\overrightarrow{\nabla K_{g}} \equiv 0$ in $M \backslash\left\{p_{1}, p_{2}, \cdots, p_{n}\right\}$. Therefore, $K_{g}$ must be a constant function in $M \backslash\left\{p_{1}, p_{2}, \cdots, p_{n}\right\}$. The theorem then holds true.

\section{Examples of HCMU metrics in $S_{2,2,2}^{2}$.}

\subsection{Calabi's examples.}

It is known that there exists a unique rotationally symmetric HCMU metric in any football if the area is given. In this subsection, we will show how $\mathrm{E}$. Calabi uses a HCMU metric in a football to construct a HCMU metric in $S_{2,2,2}^{2}$. Let $g_{p q}$ be a HCMU metric in the football $S_{\left\{\frac{1}{3}, \frac{1}{2}\right\}}$; let $p$ denote the pole with an angle of $\frac{2 \pi}{3}$ and let $q$ denote the pole with an angle of $\frac{2 \pi}{2}$. Let $S^{2}=\{$ z-plane $\} \bigcup\{z=\infty\}$; let $\zeta=e^{\frac{2 \pi \sqrt{-1}}{3}}$. Two group actions $\nu$ and $\mu$ in 


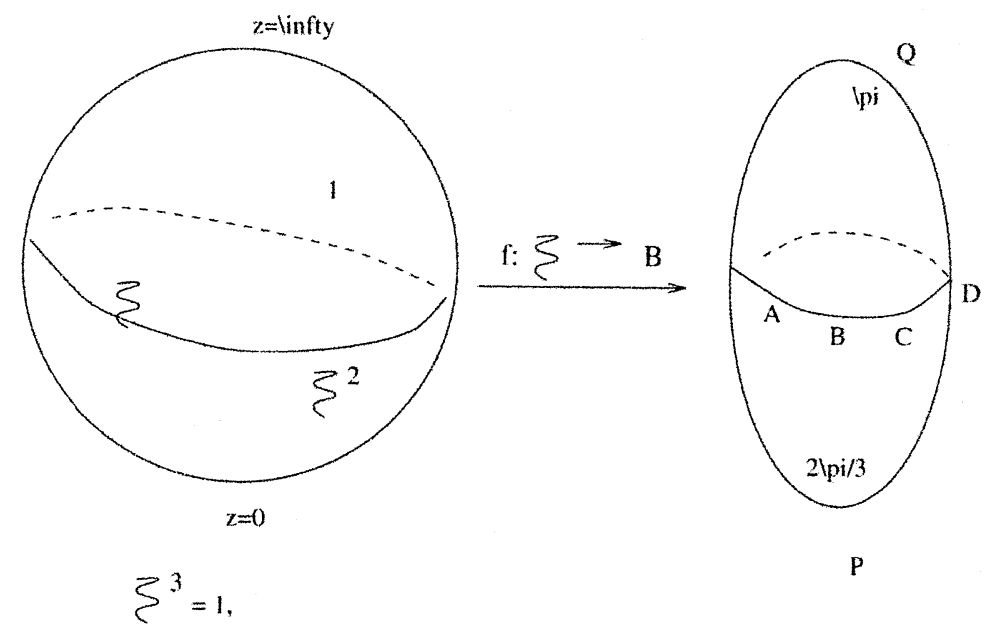

Figure 1: Calabi's metric on $S_{2,2,2}^{2}$

$S^{2}$ are defined such that:

$$
\nu(z)=\frac{1}{z}, \quad \mu(z)=z \cdot \zeta, \quad \forall z \in S^{2} .
$$

Let $G$ be the group generated by three elements: the identical map, $\nu$ and $\mu$. The order of group $G$ is 6 . $G$ acts on $S^{2}$ proper discontinuously. A generic $G$-orbit in $S^{2}$ consists of 6 distinct points. There are three singular $G-$ orbits in $S^{2}$; and these orbits are:

$$
\{0, \infty\}, \quad\left\{1, \zeta, \zeta^{2}\right\}, \quad\left\{-1,-\zeta,-\zeta^{2}\right\} .
$$

The group action induces a natural projection $\pi$ as the following:

$$
\pi: S^{2} \rightarrow S^{2} / G
$$

For any $z \in S^{2}, \pi(z)=[z]$ is the G-orbit where $z$ belongs to; and $\pi^{-1}([z])$ represents the set of points in $S^{2}$ which share a G-orbit with $z$. If $[z]$ is neither of the orbits [0], [1] and [-1], then $\pi$ is a local isometric map from a small neighborhood of $z$ to its image $[z]$. $\pi$ is a local 2-sheet covering at $[z]=[1],[-1]$; and a local 3-sheet covering at $[z]=[0]$.

Define a holomorphic map (see figure 1) $f: S^{2} / G \rightarrow S_{13,12}$ such that: $f([0])=p, f([-1])=q$, and $f([1])=B$ (any regular point) in $S_{\left\{\frac{1}{3}, \frac{1}{2}\right\}}$. This map is then uniquely determined by $f([1])$. Consider the pulled back metric $g^{0}$ in $S^{2}$ :

$$
g^{0}=\pi^{*} f^{*} g_{p q}
$$


By definition, $g^{0}$ is a HCMU metric in $S^{2}$. This metric is smooth everywhere except possibly on the following 8 points: $z=0, \infty$; and $z=-1,-\zeta,-\zeta^{2}$; and $z=1, \zeta, \zeta^{2}$. However, $g_{p q}$ has an angle of $\frac{2 \pi}{3}$ at $p$ and the projection $\pi$ has a three sheet covering at $f^{-1}(p)=[0]$. Therefore, $g^{0}$ has an angle of $2 \pi$ at the two points $z=0$ and $z=\infty$. Consequently, $g^{0}$ is smooth in these two points: $z=0$ and $z=\infty$. Similar arguments show that $g^{0}$ is smooth on the three points: $z=-1, z=-\zeta$ and $z=-\zeta^{2}$. However, $g^{0}$ has a singular angle of $4 \pi$ at the three remaining points $z=1, z=\zeta$ and $z=\zeta^{2}$. Moreover, if $f([1])$ is deformed along a "waist" line(i.e., arc "ABCD") in $S_{13,12}^{2}$, we obtain a 1-parameter local isometric deformations of the HCMU metric in $S_{2,2,2}^{2}$. This local isometric deformation preserves the global symmetric of $g^{0}$.

\subsection{Applications of Theorem 1.}

It is not known to E. Calabi then that whether the above HCMU metric is unique in $S_{2,2,2}^{2}$. However, we learn after theorem 1 that it is not unique. We have constructed another HCMU metric in $S_{2,2,2}^{2}$ which has different enengy level with Calabi's metric. To certain extent, Theorem 1 offers a "blue print" of a HCMU metric in any K-surface. Following this "blue print," one may construct a $\mathrm{HCMU}$ metric in a K-surface in a systematic fashion.

To illustrate this point, we will examine all of HCMU metrics in the K-surface $S^{2}{ }_{\{2,2,2\}}$. Let $g$ be a HCMU metric in $S^{2}{ }_{2,2,2\}}$. Following the notations in theorem $1, g$ has three singular points $\left\{p_{1}, p_{2}, p_{3}\right\}$ and $g$ has an angle of $4 \pi$ at each of these three points. Let $s$ denote the number of smooth critical points of $K_{g}$. We will divide our discussions into four cases, according to the number of the saddle points of $K_{g}$ among the three singular points of $g$.

1. If all of the singular points of $g$ are local extremal points of $K_{g}$, then $j=0$. Theorem 1 then implies :

$$
(3-0)+s=\chi\left(S^{2}\right)=2 .
$$

However $s \geq 0$ since $s$ is the number of smooth critical points of $K_{g}$. Therefore, this case is impossible.

2. If exactly two singular points of $g$ are local extremal points of $K_{g}$, then $j=1$. Theorem 1 then implies:

$$
(1-2)+(3-1)+s=2,
$$

or $s=1$. Therefore, the following three statements hold true: 
(i) $g$ must have exactly one smooth local extremal point of $K_{g}$;

(ii) $g$ must have two singular extremal points of $K_{g}$ with angles $4 \pi, 4 \pi$ respectively;

(iii) $g$ must have a singular saddle point of $K_{g}$ with an angle of $4 \pi$.

Using this information, we have explicitly constructed a sequence of surfaces $S_{2,2 \alpha, 2 \alpha}^{2}$ with HCMU metric $g_{\alpha}$ for any $\alpha>0$.

3. If exactly one singular point of $g$ is a local extremal point of $K_{g}$, then $j=2$. Theorem 1 then implies:

$$
(1-2)+(1-2)+(3-2)+s=2,
$$

or $s=3$. Therefore, the following three statements hold true:

(i) $g$ must have exactly three smooth local extremal points of $K_{g}$;

(ii) $g$ must have one singular local extremal point of $K_{g}$ with an angle of $4 \pi$; (2) $g$ must have two singular saddle points of $K_{g}$ with singular angles of $4 \pi, 4 \pi$ respectively.

Using this information, we have explicitly constructed a sequence of surfaces $S_{2,2,2}^{2}$ with HCMU metric $g$.

4. If none of the singular points of $g$ is a local extremal point of $K_{g}$, then $j=3$. Theorem 1 then implies:

$$
(1-2)+(1-2)+(1-2)+(3-3)+s=2,
$$

or $s=5$. Therefore, the following two statements hold true: (i) $g$ must have five smooth local extremal points of $K_{g}$; (ii) $g$ must have three singular saddle points of $K_{g}$ with singular angles of $4 \pi, 4 \pi, 4 \pi$ respectively. Using this information, we can construct a HCMU metric in $S_{2,2,2}^{2}$. It turns out that this is exactly the HCMU metric constructed by E.Calabi via a different method.

\subsection{Constructions of HCMU metrics.}

We will first construct a HCMU metric in $S_{2,2,2}^{2}$ as suggested in case 2 . Consider a football with angles $4 \pi \alpha(\alpha>14)$ and $\pi$ respectively at the two poles $p, q$. Equip this football with a rotationary symmetric HCMU metric. Then the curvature function decreases monotonely along any meridian from $p$ (with angle $4 \pi \alpha$ ) to $q$ (with angle $\pi$ ). Choose any point $A$ in the football 


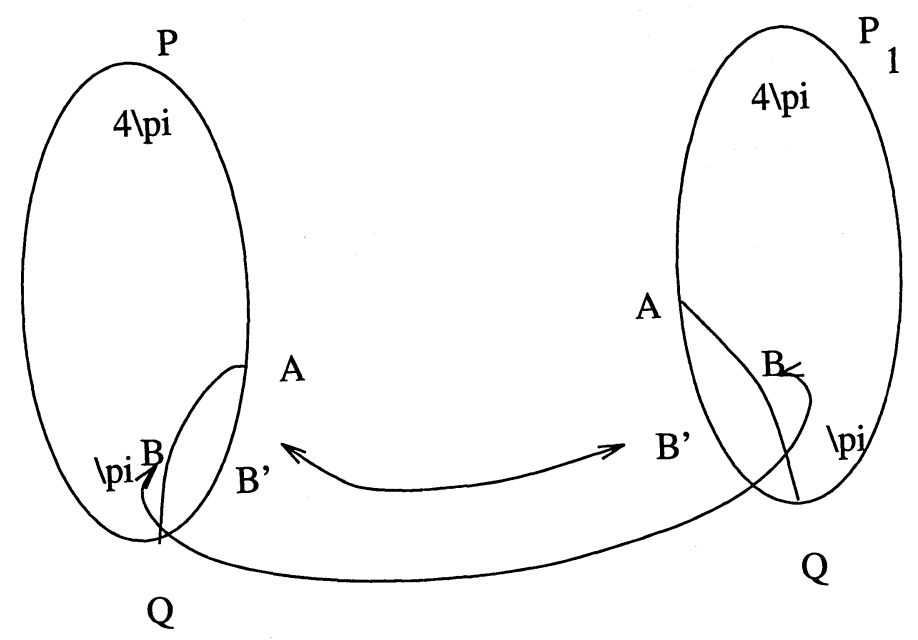

Figure 2: HCMU metric on $S_{2,2,2}^{2}$

other than $p, q$. Consider the meridian passing through $A$. Let $B$ be any point in this meridian $\widehat{q A p}$ which situated between $q$ and $A$. Now cut the football along the arc $\overrightarrow{q B A}$. The arc $\widehat{q B A}$ becomes two identical arcs: $\overrightarrow{q B A}$ and $\widehat{q B^{\prime} A}$. Construct two identical copies of this football which are cutted open exactly the same way, and re-glue the two footballs together via the open arcs (the arc $\widehat{q B A}$ in one football glues together with the arc $\widehat{q B A}$ in the other football, while the arc $\widehat{q B^{\prime} A}$ in one football glues together with the arc $\widehat{q B^{\prime} A}$ in the other football). The resulting surface is $S^{2}$, while the resulting metric has three singular points where the metric has singular angle $4 \pi \alpha, 4 \pi \alpha$, and $4 \pi$ respectively: Point $p$ and $p_{1}$ has angle $4 \pi \alpha$ respectively; point $A$ has $4 \pi$ angle; point $q$ is a smooth point after gluing. This metric is clearly HCMU, but not locally isometric to the metric constructed by $\mathrm{E}$. Calabi. In fact, These two HCMU metrics in $S_{2,2,2}^{2}$ have different energy level. Assuming the area is $2 \pi$, then the total energy of the metric given by E. Calabi is $32 \pi$, while the metrics just constructed has an energy of $52 \pi$. It remains open if calabi's metric is the real energy minimizer.

We now proceed to construct a HCMU metric as suggested in case 3. Consider a football with angles $2 \pi$ and $4 \pi 3$ respectively. As before, equip this football with a rotaionally symmetric HCMU metric. Then the curvature function strictly decreses from one pole with bigger angle to the other pole. 
Consider the double covering of this football. It is a football with angles $4 \pi$ and $8 \pi 3$ respectively. The HCMU metrics are locally identical in both footballs. Now cut both footballs into two identical pieces along two opposite meridians. We then obtain four pieces of surfaces. Reglue them together as indicated in figure 3: the arc $C E$ in 2nd football piece glue with $C E$ at the third football piece, $C F$ in 2nd football piece glue together with $C F$ in the 3rd football piece; the arc $B E$ at 3rd football piece with $B E$ at 4th football piece; etc... Clearly, the resulting surface is $S^{2}$, while the resulting metric has three singular points with angles $4 \pi, 4 \pi, 4 \pi$ : point $A, E$ and $F$ have singular angles $4 \pi$ respectively; point $B, C$ and $D$ are smooth after the gluing. This HCMU metric is also different from the one constructed by $\mathrm{E}$. Calabi. However, they have same total energy. Furthermore, there is a local isometric path from Calabi's metric to this one.

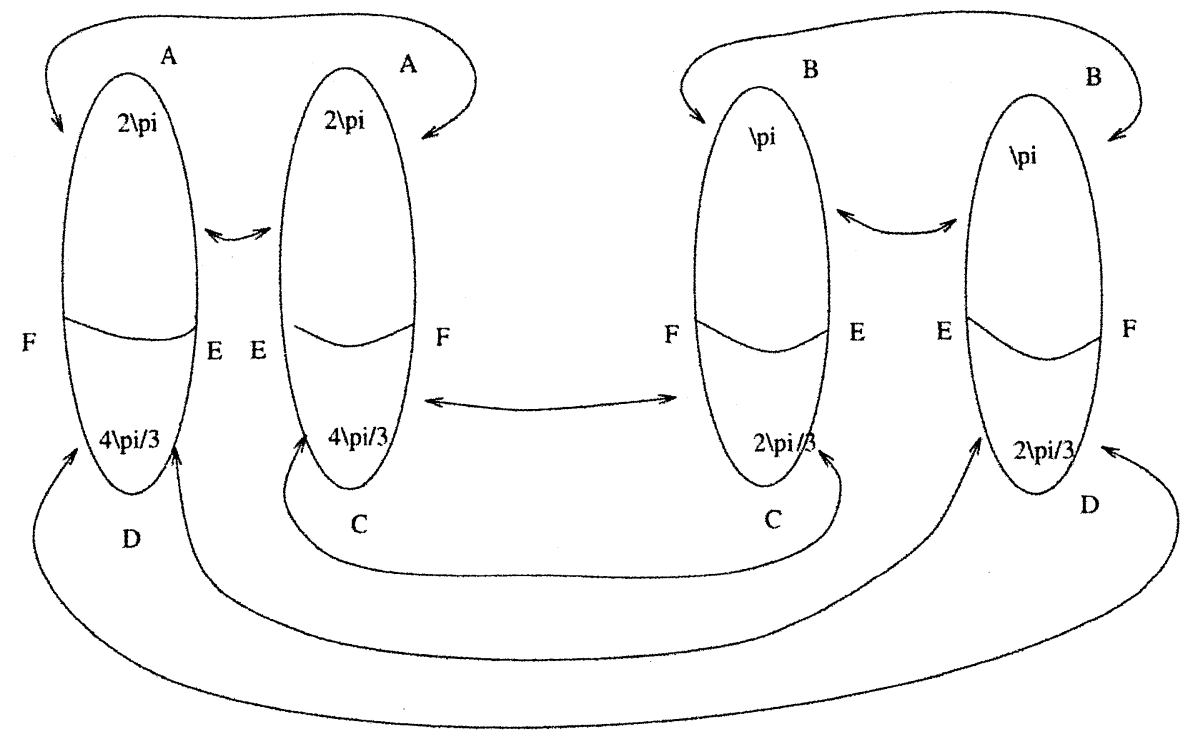

Figure 3: Example 3 of HCMU metric in $S_{2,2,2}^{2}$

We could also re-construct Calabi's metric in a similar fashion as suggested in case 4 . We will skip this part.

\section{Killing vector field of a HCMU metric.}

Unless otherwise specified; let $g$ be a HCMU metric whose curvature is not a constant function on $M_{\left\{\alpha_{1}, \alpha_{2}, \cdots, \alpha_{n}\right\}}$; let $\left\{p_{1}, p_{2}, \cdots, p_{n}\right\}$ be the set of singular 
points of $g$. We will define a special Killing vector field, and then prove that this Killing vector field has no cluster singular point.

Recall that the gradient vector field $\overrightarrow{\nabla K_{g}}$ is holomorphic since $g$ is HCMU. Hence, the real part of $\overrightarrow{\nabla K_{g}}$ must be a Killing vector field. Let $\vec{V}$ denote this Killing vector field. In a local coordinate chart, we have:

$$
\vec{V}=12\left(\sqrt{-1} K_{g}^{, z} \frac{\partial}{\partial z}-\sqrt{-1} K_{g}^{, \bar{z}} \frac{\partial}{\partial \bar{z}}\right) .
$$

Let $\operatorname{Sing}(\vec{V})$ denote the set of all singular points of vector field $\vec{V}$. It is evident that $\operatorname{Sing}(\vec{V})$ must be a subset of the union of the singular set of the metric $g$ and the critical points of $K_{g}$ in $M \backslash\left\{p_{1}, p_{2}, \cdots, p_{n}\right\}$.

$$
\text { Sing } \vec{V} \subset\left\{\text { all smooth critical points of } K_{g}\right\} \bigcup\left\{p_{1}, p_{2}, \ldots, p_{n}\right\} \text {. }
$$

We will prove that $\vec{V}$ has only a finite number of singular points. It will be proved first that any cluster point of the set of singular points of $\vec{V}$ must be a singular point of the metric as well. The strategy is to study the behavior of $\vec{V}$ at any non-cluster singular point, and eventually use this information to conclude that none of the singular points of $\vec{V}$ is a cluster point. The next proposition gives some fundmental properties of this singular set.

Proposition 2. Any cluster point of Sing $(\vec{V})$ must be a singular point of the metric $g$.

Proof. Suppose that $q \notin\left\{p_{1}, p_{2}, \cdots, p_{n}\right\}$ is a cluster point of $\operatorname{Sing}(\vec{V})$. There exists a sequence of points $\left\{A_{i}, i \in \mathbf{N}\right\} \subset \operatorname{Sing}(\vec{V})$ which converges to the point $q$. There is only a finite number of singular points for the metric $g$. The sequence of points $\left\{A_{i}\right\}$ could then be chosen so that every $A_{i}(\forall i)$ is a smooth point of $g$. Therefore, $A_{i}(\forall i)$ must be a smooth critical point of $K_{g}$. Consider a small Euclidean disk $B$ which contains $q$ in its interior. The gradient vector field $\overrightarrow{\nabla K_{g}}$ must have an infinite number of zero points in $B$. This implies that $\overrightarrow{\nabla K_{g}}$ is identically zero in $B$ since it is a holomorphic vector field. Consequently, $K_{g}$ is a constant in $B$. Theorem 4 then implies that the scalar curvature must be a constant function in $M \backslash\left\{p_{1}, p_{2}, \cdots, p_{n}\right\}$ which contradicts the initial assumption about a HCMU metric.

For any $p \in M \backslash \operatorname{Sing}(\vec{V})$, there exists a unique integral curve of $\vec{V}$ which passes $p$. Denote the complete extension of this integral curve as $C_{p}$. 
The central object of this study is this vector field and all of its integral curves. Unless otherwised claimed, the term "integral curve" means the "integral curve of $\vec{V}$." The next proposition gives some basic facts about the integral curves of $\vec{V}$.

Proposition 3. 1. Any two integral curves of $\vec{V}$ will not intersect at any point $p \notin \operatorname{Sing}(\vec{V})$. In particular, no integral curve of $\vec{V}$ has a self intersecting point in $M \backslash \operatorname{Sing}(\vec{V})$.

2. In $M \backslash \operatorname{Sing}(\vec{V})$, any integral curve of gradient flow of curvature $K_{g}$ can not intersect an integral curve of $\vec{V}$ more than once.

This proposition follows readily from the definitions of a Killing vector field and a gradient vector field of the scalar curvature function.

Lemma 1. For any $p \in M \backslash \operatorname{Sing}(\vec{V})$, if the closure of $C_{p}$ does not intersect $\operatorname{Sing}(\vec{V})$, i.e., $\overrightarrow{C_{p}} \cap \operatorname{Sing} \vec{V}=\emptyset$, then $C_{p}$ must be a closed curve.

Proof. Since $\overline{C_{p}} \cap \operatorname{Sing}(\vec{V})=\emptyset$, there exists a small number $\epsilon>0$ and an open $\epsilon$ neighborhood $\mathcal{B}^{\epsilon}$ of $\operatorname{Sing}(\vec{V})$ such that $C_{p} \subset M \backslash \mathcal{B}^{\epsilon}$. Suppose that $C_{p}$ is not a closed curve, then $C_{p}$ must have a cluster point in $M \backslash \mathcal{B}^{\epsilon}$. Let $C_{p}:[0, T) \rightarrow M \backslash \mathcal{B}^{\epsilon}(T \leq \infty)$ be a parameterization of $C$ such that:

$$
C_{p}(0)=p ; \quad C_{p}^{\prime}(t)=V\left(C_{p}(t)\right), \quad \forall t \in[0, T) .
$$

Suppose $q \subset M \backslash \mathcal{B}^{\epsilon}$ be a cluster point of $C_{p}$. Since $q$ is not a singular point of $\vec{V}$, a sequence of points $\left\{t_{i} \rightarrow T, i \in \mathbf{N}\right\}$ could be chosen such that:

$$
\lim _{i \rightarrow \infty} C_{p}\left(t_{i}\right)=q
$$

and (see figure 4)

$$
\begin{aligned}
\lim _{i \rightarrow \infty} C_{p}^{\prime}\left(t_{i}\right) & =\lim _{i \rightarrow \infty} \vec{V}\left(C_{p}\left(t_{i}\right)\right) \\
& =\vec{V}\left(\lim _{i \rightarrow \infty} C_{p}\left(t_{i}\right)\right)=\vec{V}(q) .
\end{aligned}
$$

The last equality holds true since $q$ is not a singular point of $\vec{V}$. Consider the integral curve of the gradient flow of curvature $K_{g}$ at $q$. This curve is perpendicular to $C_{q}$, and it will inevitably intersect $C_{p}$ more than once, 


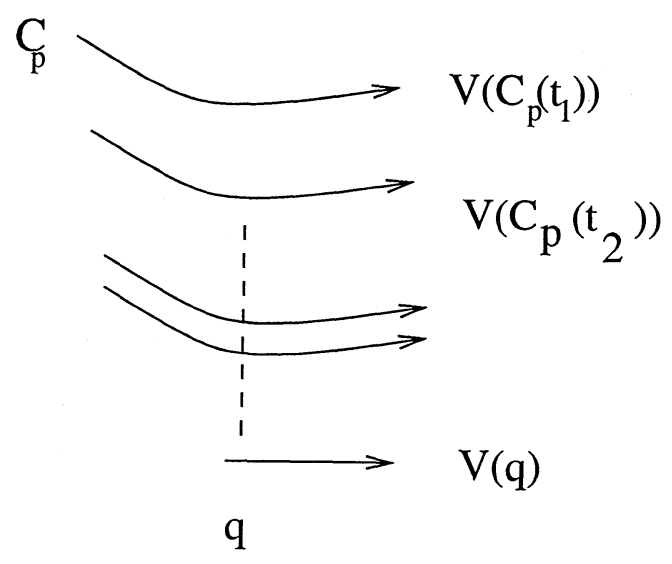

Figure 4: Closed curve

which contradicts with proposition 3.2 . Therefore, $C_{p}$ must be a closed curve. The lemma holds true.

Let $\Omega_{p}$ denote the space of all of the integral curves of $\vec{V}$ which meets $p$; let $\left|\Omega_{p}\right|$ denote the cardinality of $\Omega$. For any point $p \notin \operatorname{Sing}(\vec{V})$, there exists a unique integral curve of $\vec{V}$ which passes the point $p$. Hence $\Omega_{p}$ is well defined, and it contains two elements (one integral curve enters into $p$ and one leaves from $p$ ). However, at a singular point $p$ of $\vec{V}$, it is not clear what "an integral curve of $\vec{V}$ pass $p$ " means, since either $\vec{V}$ is not defined or $\vec{V}$ vanishes at the point $p$. To clarify this point, an integral curve $C$ of $\vec{V}$ is said to be in $\Omega_{p}(p \in \operatorname{Sing}(\vec{V}))$, if and only if there exists a sequence of points in $C$ which converges to $p$. In other words, if $C \notin \Omega_{p}$, there then exists a small disk $B_{\epsilon}$ center at $p$ such that $C \bigcap B_{\epsilon}=\emptyset$. By this definition, an integral curve $C \in \Omega_{p}$ might spiral around $p$ an infinite number of times without ever reaching $p$. However, it will be proved that this is impossible.

If $\Omega_{p} \neq \emptyset$, we want to define a partial relation on the space $\Omega_{p}$. We first define this relation under the assumption that $p$ is not a cluster point of $\operatorname{Sing}(\vec{V})$. Eventually, we will show that $\operatorname{Sing}(\vec{V})$ does not have any cluster point at all. Now choose a small enough Euclidean disk $B$, so that $p$ is the only singular point of $\vec{V}$ in $B$. According to proposition 3, any two integral curves of $\vec{V}$ can never intersect at any point other than the point $p$. Hence, modeling after the orientation of a clock, for any three points $C_{1}, C_{2}, C_{3} \in \Omega_{p}$, we define the relation $C_{1} \prec C_{2} \prec C_{3}$ if and only if $C_{1}, C_{2}$ 
and $C_{3}$ are in the anti-clockwise direction (see figure 5).

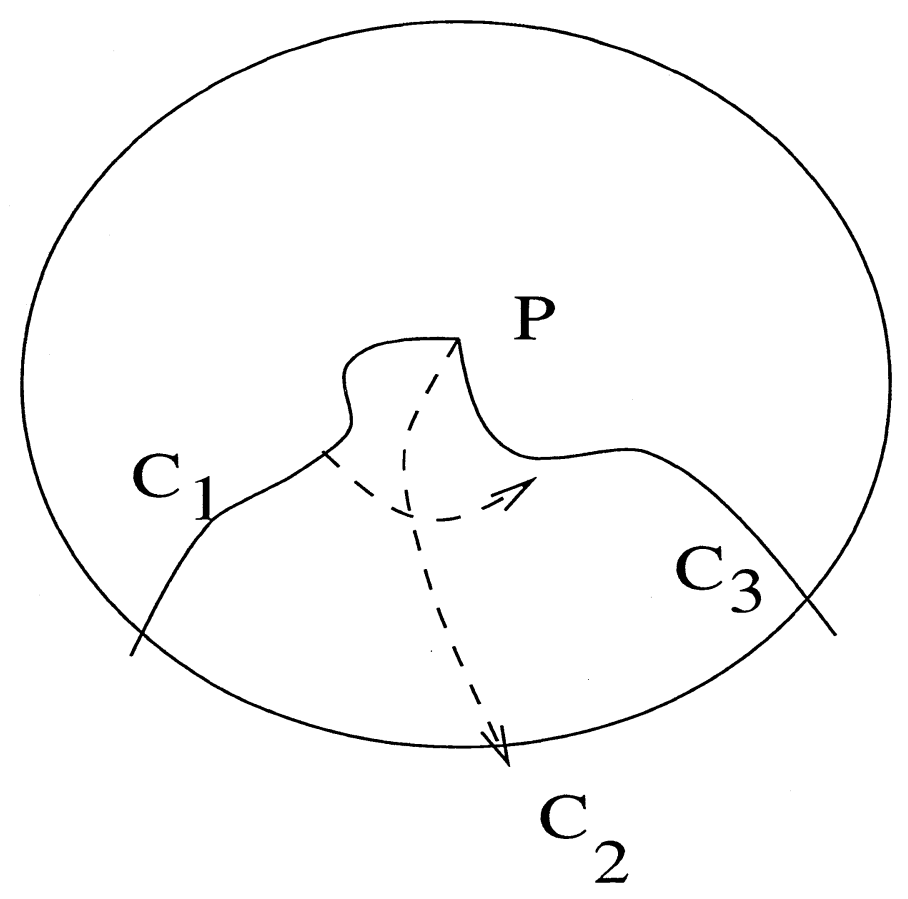

Figure 5: Orientation of integral curves

A point $p$ is said to have a dense distribution of the integral curves of $\vec{V}$ if the following condition holds true: for any two curves $C_{1}, C_{2} \in \Omega_{p}$, there always exist two additional curves $C_{3}, C_{4}$ in $\Omega_{p}$ such that the following relation holds true:

$$
C_{1} \prec C_{3} \prec C_{2} \prec C_{4} \prec C_{1} .
$$

If, for any two integral curve $C_{3}$ and $C_{4}$ such that $C_{1} \prec C_{3} \prec C_{4} \prec C_{2}$, there always exists an integral curve $C_{5}$ such that

$$
C_{1} \prec C_{3} \prec C_{5} \prec C_{4} \prec C_{2},
$$

we call that $p$ has a dense distribution bounded by $C_{1}$ and $C_{2}$. Noticed that $C_{3}$ may equal to $C_{1}$, while $C_{4}$ may equal $C_{2}$.

Lemma 2. For any point $p \in \operatorname{Sing}(\vec{V})$ which is not a cluster point of $\operatorname{Sing}(\vec{V})$, there exists no dense distribution of the integral curves of $\vec{V}$. 
Proof. Suppose there is a dense distribution of integral curves of $\vec{V}$ near $p$ and we want to draw a contradiction from this assumption. Consider two small Euclidean disks $B \subset \subset B_{1}$ satisfying the following three conditions: (i) $p \in B \subset \subset B_{1}$; (ii) $B_{1} \cap \operatorname{Sing}(\vec{V})=\{p\}$; (iii) at least two integral curves of $\vec{V}$ initiate from $\partial B$ are in $\Omega_{p}$. We claim that for any point $q \in \partial B$, then the integral curve $C_{q}$ must be in $\Omega_{p}$. Otherwise, suppose that $q$ is such a point that $C_{q} \notin \Omega_{p}$. Suppose that the parameterization of $\partial B$ as $c:[-T, T] \rightarrow B_{1}$ such that $c(0)=q$ and $c([-T, T])=\partial B$. Suppose further that $c(t)$ move in anti-clockwise direction as $t$ increases. Since $C_{q} \notin \Omega_{p}$, there exists a small neighborhood of $q$ such that any integral curve of $\vec{V}$, initiated from any point in this neighborhood, will not be in $\Omega_{p}$. Suppose that $\left[-T_{1}, T_{2}\right] \subset[-T, T]$ is the biggest interval in $[-T, T]$ such that the following two conditions hold true: (1) for any point $t \in\left(-T_{1}, T_{2}\right), C_{c(t)} \notin \Omega_{p}$; (2) Both integral curves $C_{c\left(-T_{1}\right)}$ and $C_{c\left(T_{2}\right)}$ are in $\Omega_{p}$. (see figure 6 ).



Figure 6: Dense distribution of integral curves

According to the condition (iii), the two points $c\left(-T_{1}\right)$ and $c\left(T_{2}\right)$ are different points in $\Omega_{p}$. However, the dense distribution condition of the integral curves can not be held true with respect to these two points $C_{c\left(-T_{1}\right)}$ 
and $C_{c\left(T_{2}\right)}$. Therefore, our claim must hold true. In other words, all of the integral curves initiated from $\partial D$ must be in $\Omega_{p}$. We then adjust the curve $\partial B$ slightly so that $\partial B$ transects all of the integral curves of $\vec{V}$. Consider the local maximal point of $K_{g}$ in this closed circle. There exists at least one local maximal point. Suppose that $A$ is such a point. Recall that $K_{g}$ is invariant in any integral curve of $\vec{V}$; the point $A$ is then a local maximal point of $K_{g}$. Therefore, every point in the entire integral curve $C_{A}$ is a local maximal point of $K_{g}$. This implies that the $\overrightarrow{\nabla K_{g}}$ vanishes in the integral curve $C_{A} \cdot \overrightarrow{\nabla K_{g}}$ then vanishes everywhere. Consequently, the scalar curvature is a constant function, which contradicts the initial assumption of a HCMU metric. This proves the lemma.

Lemma 3. For any point $p \in \operatorname{Sing}(\vec{V})$ which is not a cluster point of Sing $(\vec{V})$, there exists no dense distribution of the integral curves of $\vec{V}$ bounded by two integral curves $C_{1}$ and $C_{2}$

Proof. Consider a small open disk $B$ centered at $p$. Denote the sector bounded by $C_{1}$ and $C_{2}$ as $\mathcal{O}$. For any point $q \in \partial B \bigcap \mathcal{O}$, following the proof of the previous lemma, the integral curve $C_{q}$ must be in $\Omega_{p}$. According to theorem 6 in the Appendix, the curvature are asymptotically same at any two integral curves which are in $\Omega_{p}$. This imples that curvature are actually same of any two integral curve of $\Omega_{p}$ since the curvature is invariant at each integral curve. Therefore, the curvature function is constant in $\mathcal{O}$. This implies that the curvature is constant everywhere in $M$. This is a clear contradiction with the initial assumption on a HCMU metric. The lemma is then proved.

Lemma 4. If $p \in \operatorname{Sing}(\vec{V})$ is not a cluster point of $\operatorname{Sing}(\vec{V})$, then either $\Omega_{p}$ is an empty set or it has a finite number of points.

Proof. We prove this Lemma by drawing a contradiction. Suppose that this Lemma does not hold true for this point $p$. Then $\left|\Omega_{p}\right|$ must have at least a countable number of points. According to lemma 2 and 3 , there is a sequence of curves $\left\{C_{i}, i \in \mathbf{N}\right\} \subset \Omega_{p}$ such that the following two conditions hold: (1) $C_{1} \prec C_{i} \prec C_{i+1} \prec C_{1}, \forall i(\geq 2) \in \mathbf{N}$. (2) For any $i>0$, there exists no integral curve $C_{i}^{\prime} \in \Omega_{p}$ such that $C_{i} \prec C_{i}^{\prime} \prec C_{i+1}$. Any integral curve of $\vec{V}$, which is closed to $C_{i}$ and on the positive side of $C_{i}$, will always follow along $C_{i}$; this curve will then turn before hitting $p$ and go along the negative side 
of $C_{i+1}$. (see figure 7 ).

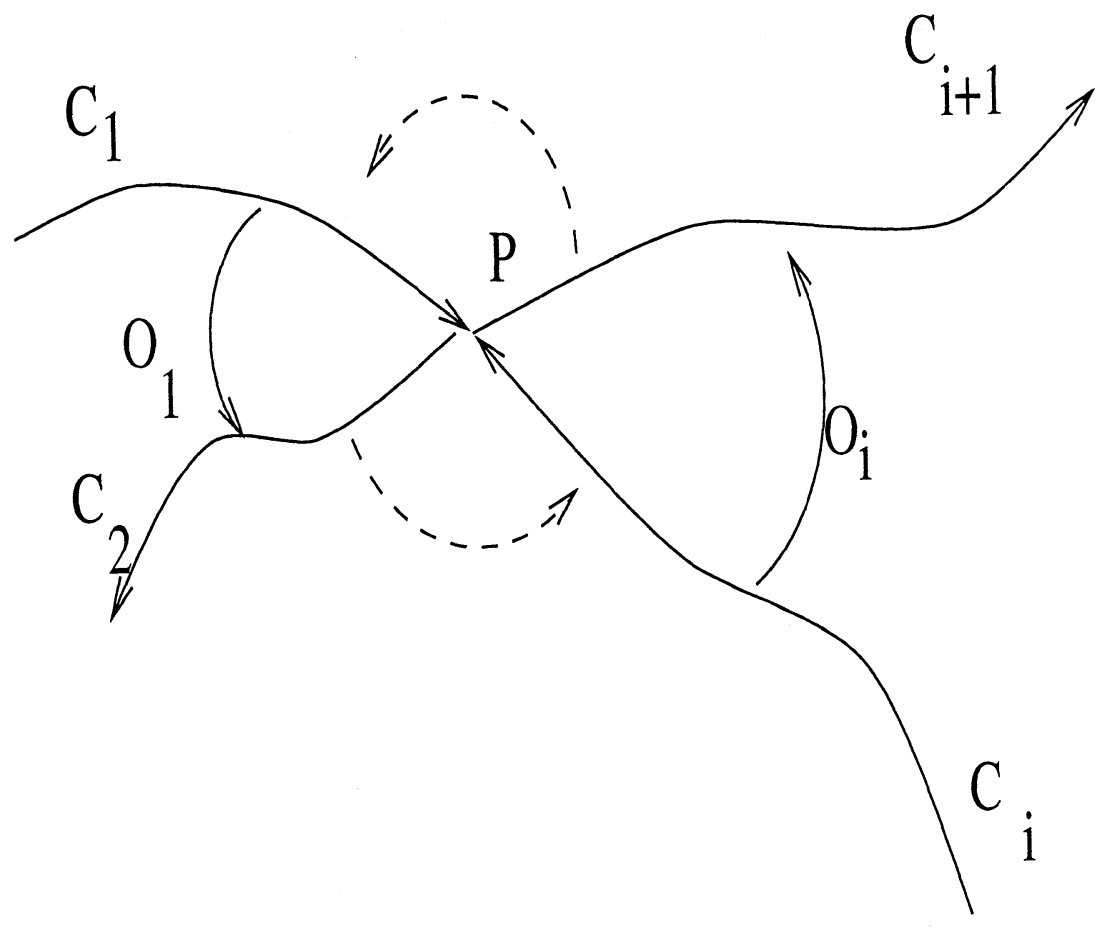

Figure 7: Singular angle is finite

Therefore, we have

$$
\angle\left(C_{i}, C_{i+1}\right)=\pi, \quad \forall i \in \mathbf{N} .
$$

The last equality implies the following equation:

$$
\text { Angle at } \begin{aligned}
p & \geq \angle\left(C_{1}, C_{m+1}\right) \\
& =\sum_{i=1}^{m} \angle\left(C_{i}, C_{i+1}\right)=m \pi \rightarrow \infty .
\end{aligned}
$$

This is also impossible because of the initial assumption that all of the singular angles are finite. Consequently, this Lemma holds true.

Lemma 5. If $p \in \operatorname{Sing}(\vec{V})$ is not a cluster point of $\operatorname{Sing}(\vec{V})$ and $\Omega_{p} \neq \emptyset$, then $\Omega_{p}$ has an even number of points. Moreover, $g$ has an angle of $\left|\Omega_{p}\right| \cdot \pi$ at the point $p$. 
Proof. Let $B$ be a small Euclidean disk containing $p$ as the only singular point of $\operatorname{Sing}(\vec{V})$. Suppose that $\left\{C_{1}, C_{2}, \cdots, C_{m}\right\}\left(m=\left|\Omega_{p}\right|\right)$ are all of the integral curves of $\vec{V}$ in $\Omega_{p}$ such that:

$$
C_{1} \prec C_{2} \prec \cdots \prec C_{m} \prec C_{1} .
$$

For any $i(1 \leq i \leq m)$, let $O(i)$ denote the small sector in $B$ bounded by $C_{i}$ and $C_{i+1}$ in the anti-clockwise direction. (Observe that $C_{m+1}=C_{1}$ and $\left.O_{m+1}=O_{1}\right)$.

$$
B=\bigcup_{i=1}^{m} O(i), \quad O_{i} \bigcap O_{i+1}=C_{i+1}, \partial O_{i} \stackrel{\text { def }}{=} C_{i} \bigcup C_{i+1} .
$$

Any integral curve of $\vec{V}$ near $C_{k}$ in $O_{k}$ will follow along $C_{k}$; this curve will then bend before hitting point $p$ and follow along $C_{k+1}$. Recall that the scalar curvature $K_{g}$ is invariant on each $C_{k}(\forall 0 \leq k \leq n)$. If $K_{g}$ increases ( resp: decreases) from $\partial O_{i}$ to the interior of $O_{i}$, then $K_{g}$ decreases (resp:increases) from $\partial O_{i+1}$ to the interior of $O_{i+1}$. (see figure 7). This statement holds true for all $i=1,2, \cdots, m$. Without the loss of generality, we suppose that $K_{g}$ increases from $\partial O_{1}$ to the interior of $O_{1}$. Then $K_{g}$ decreases from $\partial \mathrm{O}_{2}$ to the interior of $\mathrm{O}_{2}$, etc. Finally, if $m$ is an odd number, $K_{g}$ must then decrease from $\partial O_{m+1}$ to the interior of $O_{m+1}$. Recalled that $O_{m+1}=O_{1}$. This is a contradiction. Consequently, $m=\left|\Omega_{p}\right|$ must be an even number. Following the proof of of Lemma 4, we have:

$$
\angle\left(C_{i}, C_{i+1}\right)=\pi, \forall i \in[1, m] .
$$

The total angle of $g$ at the point $p$ is:

$$
\geq \sum_{i=1}^{i=m} \angle\left(C_{i}, C_{i+1}\right)=m \pi=\left|\Omega_{p}\right| \cdot \pi .
$$

Corollary 2. If $p \in \operatorname{Sing}(\vec{V})$ and $p$ is not a cluster point of Sing $(\vec{V})$; and if the prescribed singular angle of the metric $g$ at the point $p$ is not an integer multiple of $2 \pi$, then $\Omega_{p}=\emptyset$. Moreover, $p$ must be a local extremal point of $K_{g}$; and all of the integral curves of $\vec{V}$ in a small neighborhood of the point $p$ are topologically concentric circles which contain $p$ in their interiors. 
Proof. Since $p \in \operatorname{Sing}(\vec{V})$ and the angle at $p$ is not an integer multiple of $2 \pi$, lemma 5 implies that $\Omega_{p}=\emptyset$. Let $B$ be a small Euclidean disk which contains $p$ as the only singular point of $\vec{V}$. If every integral curve of $\vec{V}$ in $B$ must eventually leave $B$, there then exists at least one integral curve which meets the point $p$. This is a contradiction since we assume that $\Omega_{p}=\emptyset$. Therefore, there exists at least one integral curve of $\vec{V}$, denoted by $C_{0}$, that never leaves $B$. Since $C_{0} \notin \Omega_{p}, C_{0}$ must be a closed curve in $B$. According to Proposition $3, C_{0}$ does not have any self intersecting point in $B$. Therefore, $C_{0}$ must be a topological circle. Let $D$ denote the interior of $C_{0}$, then $p \in D$. (Otherwise there exists another singular point of $\vec{V}$ in $D \subset B$ ). Any integral curve of $\vec{V}$ in $D$ must be topologically a circle since it can not intesect with $\partial D$. For the same reason as $C_{0}$, these circles must contain $p$ in their interiors. Therefore, these circles are topologically concentric. Thus, $g$ is rotationary symmetric in $D \backslash\{0\}$. Since the curvature $K_{g}$ is not a constant in $D \backslash\{0\}$, then $K_{g}$ achieve either local maximum or minimum at $p$, following the theorem below.

Theorem 5. If $g$ is a rotationary symmetric HCMU metric in a punctured disk $D \backslash\{0\}$ with finite area, then $K_{g}$ continuous at $z=0$. Moreover, if $K_{g}$ is not a constant, then it achieves either local maximum or local minimum at $z=0$.

Proof. Let $u=-\ln |z|$, then any rotationary symmetric metric $g$ in $D \backslash\{0\}$ can be expressed as a metric in $S^{1} \times[b, \infty)$ for some $b>0$ :

$$
g=e^{2 \varphi(u)}\left(d u^{2}+d \theta^{2}\right) .
$$

The metric has finite area if

$$
\int_{b}^{\infty} e^{2 \varphi(u)} d u<\infty
$$

The metric is HCMU if

$$
\frac{\partial^{2} K_{g}}{\partial u^{2}}-2 \cdot \frac{\partial K_{g}}{\partial u} \cdot \frac{\partial \varphi(u)}{\partial u}=0,
$$

or

$$
\frac{\partial}{\partial u}\left(e^{-2 \varphi} \cdot \frac{\partial K_{g}}{\partial u}\right)=0 .
$$

Therefore, $K_{g}^{\prime}(u)=c \cdot e^{2 \varphi}$ for some constant $c$. If $c=0$, then $K_{g}^{\prime} \equiv 0$ and $K_{g}$ is a constant for all $u$. If $c>0$, then $K_{g}$ strictly increased when $u \rightarrow \infty$. 
Thus, $K_{g}$ achieves local maximum at $z=0$. Similarly, if $c<0, K_{g}$ achieves local minimum at $z=0$.

We can define

$$
K_{g}(u=\infty)=K_{g}(b)+\int_{b}^{\infty} K_{g}^{\prime}(u) d u=K_{g}(b)+c \cdot \int_{b}^{\infty} e^{2 \varphi(u)} d u .
$$

Clearly, $K_{g}$ is then finite and continuous at $z=0$.

Corollary 3. Every critical point of the scalar curvature $K_{g}$ on $M \backslash$ $\left\{p_{1}, p_{2}, \ldots, p_{n}\right\}$ must be a local extremal point of $K_{g}$.

Proof. We prove this corollary by drawing a contradiction. Suppose that $p$ is a smooth saddle point of $K_{g}$. Then $p$ is a singular point of $\vec{V}$. If $\Omega_{p}=\emptyset$, corollary 2 then implies that $p$ must be a local extremal point of $K_{g}$. This contradicts the initial assumption about $p$. Therefore, $\Omega_{p} \neq \emptyset$. According to Lemma $5,\left|\Omega_{p}\right|$ must be a even number. If $\left|\Omega_{p}\right|=2$, then $p$ is not a saddle point of $K_{g}$. Hence $\left|\Omega_{p}\right| \geq 4$ and the metric $g$ has an angle of $\left|\Omega_{p}\right| \cdot \pi \geq 4 \pi$ at $p$, However, $p$ is a smooth point of $g$. This is a contradiction. Consequently, this corollary must hold true.

Proposition 4. If $C$ is an integral curve of $\vec{V}$ which bounds a topological disk $D$ in $M \backslash\left\{p_{1}, p_{2}, \cdots, p_{n}\right\}$, and if $C$ contains no singular point of $\vec{V}$, there then exists exactly one singular point of $\vec{V}$ in $D$ which is a smooth, local extremal point of $K_{g}$. Moreover, for any Euclidean disk $D_{1}$ which contains $D$ in its interior, there exists a maximal topological disk $\tilde{D} \subset D_{1}$ such that the following two statements hold true: (1) $\partial \tilde{D}$ is an integral curve of $V$, it either tangents with $\partial D_{1}$ or passes a singular point of $\vec{V}$ in $D_{1}$. (2) All of the integral curves of $\vec{V}$ in the interior of $\tilde{D}$ are topologically concentric circles

Proof. Since $D \subset M \backslash\left\{p_{1}, p_{2}, \cdots, p_{n}\right\}$, there is no cluster point of $\operatorname{Sing}(\vec{V})$ in $D$. There are at most a finite number of singular points of $\vec{V}$ in $D$. Suppose that there are $m$ such points and suppose that they are $\left\{q_{1}, q_{2}, \cdots q_{m}\right\} \subset$ $\operatorname{Sing}(\vec{V}) \cap D$. According to corollary 3 , any $q_{i}(1 \leq i \leq m)$ must be a local extremal point of $K_{g}$; all of the integral curves of $\vec{V}$ near $q_{i}$ must be topologically concentric circles. Consider the index of the the vector field $\vec{V}$ in $\underline{D}$. Since $\vec{V}$ agrees with the tangential direction of $\partial D$, the total index of $\vec{V}$ in $D$ must be 1 . However, the index of $\vec{V}$ at each singular point is 1 . 
Therefore, there is exactly one and only one singular point in $D$. The proof for the rest of this proposition is clear.

Now proposition 3.1 could be restated as following:

Proposition 3.1. Any two integral curves of $\vec{V}$ will not intersect at any point $p \notin\left\{p_{1}, p_{2}, \cdots, p_{n}\right\}$.

Proof. Let $C_{1}$ and $C_{2}$ be two integral curves of $\vec{V}$. Suppose that they intersect at a point $q \notin\left\{p_{1}, p_{2}, \cdots, p_{n}\right\}$. Then $q$ is a smooth critical point of $K_{g}$. Therefore, all of the integral curves of $\vec{V}$ near $q$ must be topologically concentric circles. This is an apparent contradiction. Hence this proposition holds true.

Lemma 6. Any point $p \in\left\{p_{1}, p_{2}, \cdots, p_{n}\right\}$ is not a cluster point of $\operatorname{Sing}(\vec{V})$.

Proof. Suppose that $p$ is a cluster point of $\operatorname{Sing}(\vec{V})$. Choose a small Euclidean ball $B$ such that the following two statements hold true: (1) $B$ contains $p$ as the only singular point of the metric $g ;(2) \partial B \bigcap \operatorname{Sing}(\vec{V})=\emptyset$. Suppose that $\left\{q_{i}, i \in \mathbf{N}\right\}$ is a sequence of points in $\operatorname{Sing}(\vec{V}) \backslash\left\{p_{1}, p_{2}, \cdots, p_{n}\right\}$ such that the following holds true:

$$
\lim _{i \rightarrow \infty} q_{i}=p ; \quad\left\{q_{i}, i \in \mathbf{N}\right\} \subset B \bigcap \operatorname{Sing}(\vec{V}) .
$$

For any $i \in \mathbf{N}, q_{i}$ is a smooth critical point of $K_{g}$. Therefore, all of the integral curves of $\vec{V}$ near $q_{i}$ are topologically concentric circles. According to proposition 4 , there exists a maximal disk $D_{i} \subset B$ such that $q_{i} \in D_{i}$ and all of the integral curves of $\vec{V}$ are topologically concentric circles. Consider the sequence of maximal disks $\left\{D_{i}, i \in \mathbf{N}\right\}$ in $B$. For any $i \neq j, D_{i}$ and $D_{j}$ can not intersect at any point other than $p$. Therefore, we have either $D_{i} \cap D_{j}=\{p\}$ or $D_{i} \cap D_{j}=\emptyset$. Either there is an infinite number of pairs $(i, j)$ so that $D_{i} \cap D_{i}=\{p\}$, or there is an infinite number of pairs $(i, j)$ so that $D_{i} \cap D_{j}=\emptyset$. In the first case, (see figure 8), without loss of generality, we may assume that $D_{i} \cap D_{j}=\{p\}, \forall i \neq j$. 

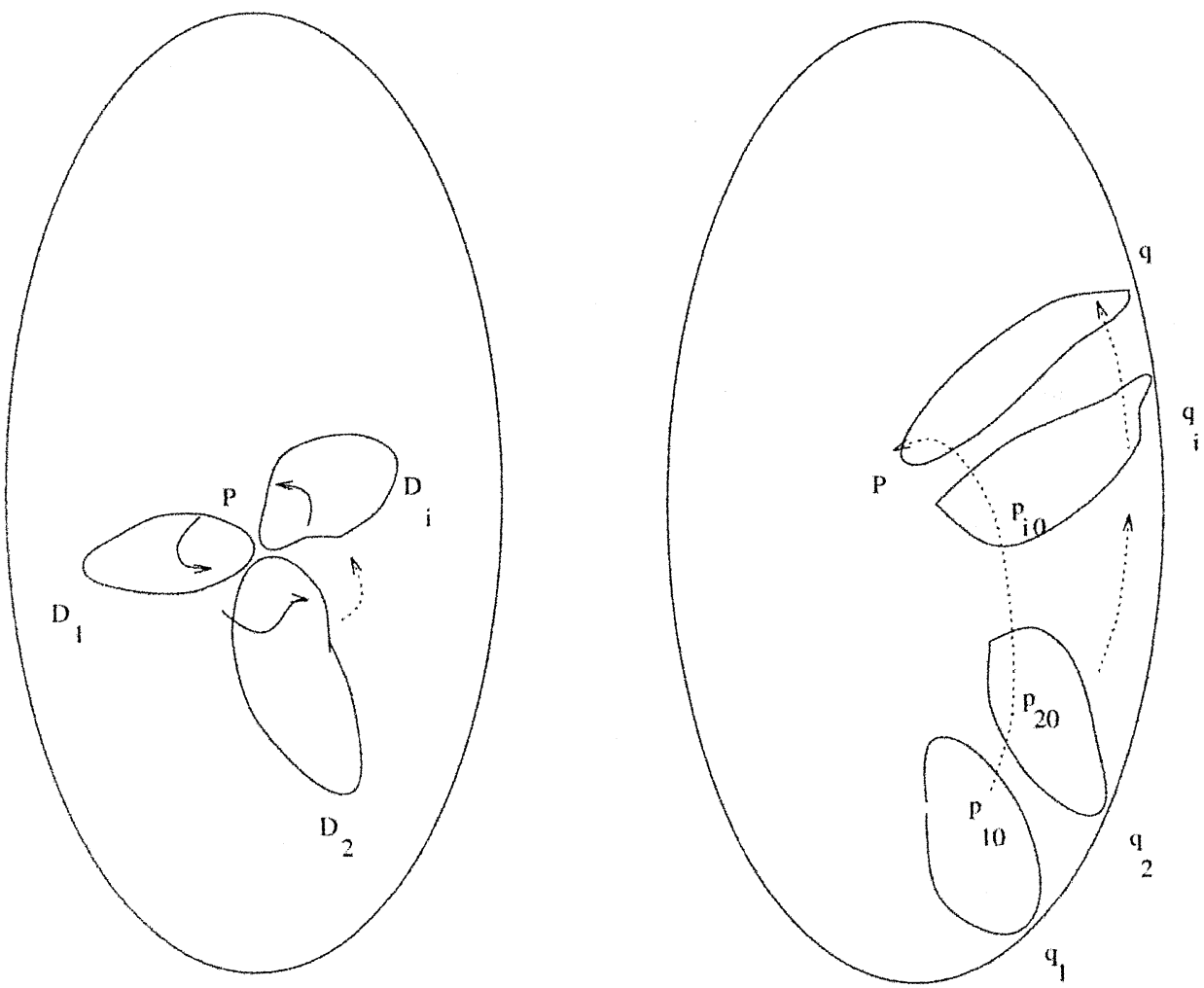

Figure 8: No cluster point

For any $i \geq 1$, consider the section bounded by $D_{i}$ at $p$. The interior angle at $p$ bounded $\partial D_{i}$ (towards the interior of $D_{i}$ ) is $\pi$. However, there is an infinite number of such interior angles at $p$. Therefore, the singular angle at $p$ must surpass $\infty$, which contradicts the initial assumption that all of the singular angles are finite. Therefore, the first case is not true. Consider the second case now. We may assume that $D_{i} \cap D_{j}=\emptyset, \forall i \neq j$. Since $D_{i}(\forall i \geq 1)$ is a maximal disk in $B$, there then exists a point $q_{i} \in \partial D_{i} \cap \partial B$. Since $\left\{q_{i}, i \in \mathbf{N}\right\}$ is a sequence of points in a Euclidean circle $\partial B$, there must exist a cluster point $q \in \partial B$ such that $q_{i} \rightarrow q$. The integral curves of $\vec{V}$ at $\left\{q_{i}\right\}$ must converge to the integral curve of $\vec{V}$ at $q$ since $\left\{q, q_{i}, i \in \mathbf{N}\right\}$ are smooth points of $\vec{V}$. On the other hand, all of the disks $D_{i}$ are in $B$. Therefore, the Euclidean area of $D_{i}$ must converge to 0 . let $p_{i 0}$ denote the unique singular point of $\vec{V}$ in the interior of $D_{i}$. Observe that any point other than $p$ is not a cluster point of $\operatorname{Sing}(\vec{V})$. Passing to a subsequence if 
necessary, we may assume that $p_{i 0} \rightarrow p$. Consequently, the disk $D_{i}$ converges to a double curve from $q$ to $p$. Thus the geodesic curvature of $\partial D_{i}$ at $q_{i}$ must converge to $\infty$ as $q_{i} \rightarrow q$. This is a contradiction since $q$ is not a singular point of $\vec{V}$. Therefore, $p$ is not a cluster point. Thus, the Lemma is proved.

\section{Proof of Theorem 1 and Theorem 2.}

For reader's convenience, we copy the statements of Theorem 1 and Theorem 2 here.

Theorem 1. Let $g$ be a HCMU metric in a K-surface $M \backslash\left\{\alpha_{1}, \alpha_{2}, \cdots, \alpha_{n}\right\}$. Then the Euler-Lagrange character of the underlying surface should be determined by

$$
\chi(M)=\sum_{i=1}^{j}\left(1-\alpha_{i}\right)+(n-j)+s
$$

where $s$ is the mumber of critical points of the curvaure $K_{g}$ (excluding the singular points of $g)$. Here we assume that $\alpha_{1}, \alpha_{2}, \ldots . \alpha_{k}(0 \leq k \leq n)$ are the only integers in the set of prescribed angles $\left\{\alpha_{1}, \alpha_{2}, \cdots, \alpha_{n}\right\}$; and assume that $\left\{p_{j+1}, \cdots, p_{k}\right\}$ are the only local extremal points of $K_{g}$ in the set of singular points $\left\{p_{j}, 0 \leq j \leq k\right\}$.

Theorem 2. If none of the prescribed angles in a $K$-surface is an integer multiple of $2 \pi$, this surface supports a HCMU metric if and only it is a football with two different angles at the two poles. Furthermore, any HCMU metric in these footballs must be rotationally symmetric and uniquely determined by the area.

Proof of theorem 1. We first decompose $\operatorname{Sing}(\vec{V})$ into two parts: $\operatorname{Sing}(\vec{V})=\mathcal{S}_{1} \bigcup \mathcal{S}_{2}$ where

$$
\mathcal{S}_{1} \stackrel{\text { def }}{=}\left\{p \in \operatorname{sing}(\vec{V}): \Omega_{p}=\emptyset\right\}, \quad \mathcal{S}_{2} \stackrel{\text { def }}{=}\left\{p \in \operatorname{Sing}(\vec{V}): \Omega_{p} \neq \emptyset\right\} .
$$

For any point $p \in \mathcal{S}_{2}$, then $\left|\Omega_{p}\right|=2 \cdot l \geq 4$. Lemma 5 implies that $g$ has a singular angle of $2 \pi \cdot l$ at the point $p$. Therefore, $p$ is a singular point of $g$ and $p \in\left\{p_{1}, p_{2}, \cdots, p_{k}\right\}$. Consequently, we have: $\mathcal{S}_{2} \subset\left\{p_{1}, p_{2}, \cdots, p_{k}\right\}$. For convenience, suppose that $\mathcal{S}_{2}=\left\{p_{1}, p_{2}, \cdots, p_{j}\right\}(j \leq k)$. For any $1 \leq$ $i \leq j, g$ has a singular angle of $2 \pi \alpha_{i}$ at $p_{i}$. Since $\Omega_{p_{i}} \neq \emptyset$, Lemma 5 then 
implies that $\left|\Omega_{p_{i}}\right|=2 \alpha_{i}$. The indexes of vector field $\vec{V}$ at these points must then be:

$$
\operatorname{index}_{p_{i}}(\vec{V})=1-\alpha_{i}, \forall 1 \leq i \leq j
$$

Recall that

$$
\left\{p_{1}, p_{2}, \cdots, p_{n}\right\} \subset \operatorname{Sing}(\vec{V})=\mathcal{S}_{1} \bigcup \mathcal{S}_{2}
$$

and

$$
\mathcal{S}_{2}=\left\{p_{1}, p_{2}, \cdots, p_{j}\right\}
$$

Therefore, $\left\{p_{i}, j<i \leq n\right\} \subset \mathcal{S}_{1}$. According to the definition of $\mathcal{S}_{1}$, the indexes of vector field $\vec{V}$ at these points are:

$$
\operatorname{index}_{p_{i}}(\vec{V})=+1, \forall j<i \leq n .
$$

Observe that $\left\{q_{i}, \forall 1 \leq i \leq s\right\}$ are all smooth critical points of $K_{g}$. Thus we have:

$$
\mathcal{S}_{1}=\left\{p_{j+1}, \cdots, p_{n}\right\} \bigcup\left\{q_{1}, q_{2}, \cdots, q_{s}\right\}
$$

According to Corollary 3 , all of these points $\left\{q_{1}, q_{2}, \cdots, q_{s}\right\}$ are local extremal points of $K_{g}$. The indexes of the vector field $\vec{V}$ at these points must then be

$$
\operatorname{index}_{q_{i}}(\vec{V})=+1, \forall 1 \leq i \leq s .
$$

The total index of vector field $\vec{V}$ must equal with Euler-Lagrange character of $M$, thus

$$
\sum_{i=1}^{s} \operatorname{index}_{q_{i}}(\vec{V})+\sum_{i=j+1}^{n} \operatorname{index}_{p_{i}}(\vec{V})+\sum_{i=1}^{j} \operatorname{index}_{p_{i}}(\vec{V})=\chi(M)
$$

or

$$
s+(n-j)+\sum_{i=1}^{j}\left(1-\alpha_{i}\right)=\chi(M) .
$$

This proves the theorem. 
Proof. Now we want to prove the corollary 1 of this theorem. Recall that for any $i(1 \leq i \leq k)$, we have $\alpha_{i} \geq 2$. The above equation then implies:

$$
\begin{aligned}
\sum_{i=1}^{k}\left(1-\alpha_{i}\right)+(n-k) & \leq \sum_{i=1}^{j}\left(1-\alpha_{i}\right)+(n-j) \\
& \leq \sum_{i=1}^{j}\left(1-\alpha_{i}\right)+(n-j)+s=\chi(M) .
\end{aligned}
$$

corollary 1.4 is then proved. Corollary 1.1 and 1.2 follow from Corollary 2, Corollary 1.3 follows from Corollary 3 .

Proof of theorem 2. Let $g$ be a HCMU metric in $M \backslash\left\{\alpha_{1}, \alpha_{2}, \cdots, \alpha_{n}\right\}$. We will follow the notations of theorem 1 . Observe that none of the prescribed singular angles is an integer multiple of $2 \pi$. Corollary 1 then implies:

$$
0+(n-0) \leq \chi(M) .
$$

Thus, $\chi(M)=2$, or $M=S^{2}$, and $n \leq 2$. The K-surface must be one of the following three surfaces: (1) a 2-sphere $S^{2}$ if $n=0$; (2) a tear drop $S_{\alpha}^{2}, \alpha>0, \alpha \neq 1$ if $n=1$; (3) a football $S_{\left\{\alpha_{1}, \alpha_{2}\right\}}^{2}$ if $n=2$. Theorem 2 then implies:

$$
0+(n-0)+s=\chi(M)=2,
$$

or $s+n=2$. The following two statements hold true: (1) $g$ has exactly two singular points of $\vec{V} ;(2)$ both of these two singular points are local extremal point of $K_{g}$ : one is a local maximal point and the other is a local minimal point. According to corollary 3, all of the integral curves of the $\vec{V}$ must be topologically concentric circles near each extremal point. This consequently forces all of the integral curves of $\vec{V}$ to be concentric circles as well. Therefore, the metric $g$ is rotationally symmetric. The scalar curvature function of $g$ must be a constant function if $M=S^{2}$ and $M=$ $S_{\alpha_{1}, \alpha_{2}}^{2}\left(\alpha_{1}=\alpha_{2}\right)$. That contradicts the initial assumption about a HCMU metric. Consequently, $M=S_{\alpha}^{2}(\alpha \neq 1)$ and $M=S_{\alpha_{1}, \alpha_{2}}^{2}\left(\alpha_{1} \neq \alpha_{2}\right)$. In [15], it is shown that a HCMU metric in $S_{\alpha}^{2}(\alpha \neq 1)$ or $M=S_{\alpha_{1}, \alpha_{2}}^{2}\left(\alpha_{1} \neq \alpha_{2}\right)$ must be uniquely determined by the area. These HCMu metrics had been explicitly constructed in [15]. 


\section{Appendix.}

In this section, we consider the oscillation of metrics and its scalar curvature near a singular point in a sequence of concentric circles centered at the singular point. For any function $\varphi(x)$ in an open set of $x=0$, define the oscillation function as:

$$
\mathcal{O}(\varphi(x))=\max _{|z|=|y|=|x|}|\varphi(z)-\varphi(y)| .
$$

Suppose $z=0$ is a singular point of metrics with a conical singular angle, and suppose the metric can be expressed locally as:

$$
g=e^{2 \varphi}|d z|^{2} .
$$

Follow the definition of a conical singular angle, then $\lim _{x \rightarrow 0} \mathcal{O}(\varphi(x))=0$. We want to show that the scalar curvature function $K=-\frac{\Delta \varphi}{e^{2 \varphi}}$ has the same property near the singular point:

Theorem 6. For any HCMU metric, the curvature function is also asymtopically equal along every direction. In other words, $\lim _{z \rightarrow 0} \mathcal{O}(K(z))=0$, where $z=0$ is a singular point of the metric $g$ with a conical angle.

Let $D$ be an open unit disk centered at $z=0$. Let $u=-\ln r=$ $-\ln \sqrt{x^{2}+y^{2}}$ and $\theta=\tan ^{-1} y x$. The domain $D \backslash\{0\}$ becomes an infinite cylinder $\{(u, \theta) \mid 0 \leq u \leq \infty,-\pi \leq \theta \leq \pi\}$ via this transformation. Let $\psi(u, \theta)=\varphi\left(e^{-u} \cos \theta, e^{-u} \sin \theta\right)-u$. Then $\psi$ satisfies the following inequalities:

$$
\begin{cases}\left.\int_{0}^{\infty} \int_{0}^{2 \pi} \frac{(}{\triangle_{u, \theta}} \psi\right)^{2} e^{2 \psi} d \theta d u & \leq C_{2} \\ \int_{0}^{\infty} \int_{0}^{2 \pi} e^{2 \psi} d \theta d u & \leq C_{1}\end{cases}
$$

where $\triangle_{u, \theta}=\frac{\partial^{2}}{\partial u^{2}}+\frac{\partial^{2}}{\partial \theta^{2}}$. According to theroem 2.2 in [16], $\psi(u) \rightarrow-\infty$ uniformly. Consider $\tilde{D}=(-1,1) \times(0,2 \pi)$. For any sequence of numbers $u_{i} \rightarrow \infty$, we define

$$
\begin{gathered}
\varphi_{i}(u, \theta)=\psi\left(u+u_{i}, \theta\right), \forall i \in \mathbf{N} \\
K_{i}(u, \theta)=K\left(u+u_{i}, \theta\right)=-{\frac{\triangle_{u, \theta} \psi\left(u+u_{i}, \theta\right)}{e}}^{2 \psi\left(u+u_{i}, \theta\right)} .
\end{gathered}
$$


Lemma 7. For any $u_{i} \rightarrow \infty$, then $\left\|\varphi_{i}\right\|_{H^{2,2}(\tilde{D})} \rightarrow 0$. In particularly, $\left\|\nabla \varphi_{i}\right\|_{L^{p}(\tilde{D})} \rightarrow 0$, for any $p>1$.

Proof. Consider the curvature equation:

$$
-\triangle_{u, \theta} \varphi_{i}=K_{i} \cdot e^{2 \varphi_{i}} .
$$

Observe that $\int_{\tilde{D}} K_{i}^{2} e^{2 \varphi_{i}} d u d \theta<C$ and $\varphi_{i} \rightarrow-\infty$, we have:

$$
\left\|\triangle_{u, \theta} \varphi_{i}\right\|_{L^{2}(\tilde{D})} \rightarrow 0 .
$$

Since the oscillation of $\varphi_{i}$ approaches 0 as $i \rightarrow \infty$,

$$
\left\|\varphi_{i}\right\|_{H^{2,2}(\tilde{D})} \rightarrow 0
$$

This in turn implies that:

$$
\left\|\nabla \varphi_{i}\right\|_{L^{p}(\tilde{D})} \rightarrow 0, \quad \forall p>1 .
$$

Lemma 8. Near the singular point, the curvature function satisfyies

$$
\left\|\nabla^{2} K_{i}\right\|_{L^{2}(\tilde{D})} \rightarrow 0 \text {. }
$$

Proof. For any sub-domain $\left[u_{1}, u_{2}\right] \times S^{1}$, integrate both sides of the EulerLagrange-Lagrange equation:

$$
\triangle_{u, \theta} K+K^{2} \cdot e^{2 \psi}=C \cdot e^{2 \psi}
$$

over this domain, we obtain:

$$
\begin{aligned}
\int_{o}^{2 \pi} \frac{\partial K}{\partial u}\left(u_{1}, \theta\right) d \theta-\int_{o}^{2 \pi} \frac{\partial K}{\partial u}\left(u_{2}, \theta\right) d \theta & \\
& =\int_{0}^{2 \pi} \int_{u_{2}}^{u_{1}}\left(-K^{2} \cdot e^{2 \psi}+C \cdot e^{2 \psi}\right) d u d \theta .
\end{aligned}
$$

The right side is stictly bounded for all $u_{1}$ and $u_{2}$, and goes to zero if both $u_{1}$ and $u_{2}$ approach $\infty$. Thus,

$$
\lim _{u \rightarrow \infty} \int_{0}^{2 \pi} \frac{\partial K}{\partial u}\left(u_{1}, \theta\right) d \theta=c
$$


for some constants $c$. Let us define

$$
c_{i}=\int_{\tilde{D}} \frac{\partial K_{i}}{\partial u}(u, \theta) d \theta d u
$$

Then,

$$
\lim _{i \rightarrow \infty} c_{i}=c
$$

Clearly,

$$
\int_{\tilde{D}} \frac{\partial K_{i}}{\partial \theta}(u, \theta) d \theta d u=0
$$

Therefore,

$$
\left\|\frac{\partial K_{i}}{\partial \theta}\right\|_{L^{4}(\tilde{D})} \leq C \cdot\left\|\nabla^{2} K_{i}\right\|_{L^{2}(\tilde{D})}
$$

and

$$
\left\|\frac{\partial K_{i}}{\partial u}\right\|_{L^{4}(\tilde{D})} \leq C \cdot\left\|\nabla^{2} K_{i}\right\|_{L^{2}(\tilde{D})}+C
$$

for some constants $C$ independent of $i$.

On the other hand, we have:

$$
K_{i, w w}=\frac{\partial^{2} K_{i}}{\partial w^{2}}-2 \frac{\partial K_{i}}{\partial w} \cdot \frac{\partial \varphi_{i}(w, \bar{w})}{\partial w}=0
$$

where $w=u+\sqrt{-1} \theta$. Therefore,

$$
\begin{aligned}
\left\|\frac{\partial^{2} K_{i}}{\partial w^{2}}\right\|_{L^{2}(\tilde{D})} & \leq 4 \cdot \sqrt{\left\|\nabla K_{i}\right\|_{L^{4}(\tilde{D})} \cdot\left\|\nabla \varphi_{i}\right\|_{L^{4}(\tilde{D})}} \\
& \leq 4 \cdot \epsilon_{i} \cdot\left(C+C \cdot\left\|\nabla^{2} K_{i}\right\|_{L^{2}(\tilde{D})}^{12}\right)
\end{aligned}
$$

for some $\epsilon_{i} \rightarrow 0$ as $i \rightarrow \infty$.

From the Euler-Lagrange equation, since the conformal parameter function $\varphi_{i}$ is asymptotically rotationally symmetric, we can show that

$$
\lim _{i \rightarrow \infty} K_{i}(u, \theta) \cdot e^{\varphi_{i}(u, \theta)}=0 .
$$

Following from this estimate, then we have $\left\|\triangle K_{i}\right\|_{L^{2}(\tilde{D})} \rightarrow 0$. Equation (6.2) then implies

$$
\left\|\nabla^{2} K_{i}\right\|_{L^{2}(\tilde{D})} \leq \epsilon_{i}^{\prime}
$$


for some $\epsilon_{i}^{\prime} \rightarrow 0$. That implies that

$$
\left\|\nabla^{2} K_{i}\right\|_{L^{2}(\tilde{D})} \rightarrow 0
$$

as $i \rightarrow \infty$.

Proof of Theorem 6. The previous Lemma implies

$$
\left\|\nabla \frac{\partial K_{i}}{\partial \theta}\right\|_{L^{2}(\tilde{D})} \rightarrow 0
$$

and

$$
\left\|\frac{\partial K_{i}}{\partial \theta}\right\|_{L^{p}(\tilde{D})} \rightarrow 0, \quad \forall p>1
$$

Thus theorem 6 readily follows from here.

\section{References.}

[1] H. Brezis and F. Merle, Uniform estimates and blow up behavior for solutions of $-\Delta u=V(x) e^{u}$ in two dimension, Comm. Partial Differential Equation 16 (1991), 1223-1253.

[2] E. Calabi, Extremal Kähler metrics, ii, Differential geometry and Complex analysis, Springer, 1985, 96-114.

[3] , Constant curvature metric with three conical singularities in $S^{2}, 1993$, private communication.

[4] Extremal metric in $S^{2}$ with three singular points with angle $4 \pi$ at each point, 1993, private communication.

[5] A. Chang and P. Yang, Conformal deformations of metrics on $S^{2}$, J. Diff. Geom. 27 (1988), 256-296.

[6] W. Chen and C. Li, Classification of solutions of some nonlinear elliptic equations, Duke Math. J. 63 (1991), 173-188.

[7] C.Bavard et P. Pansu, Sur l'space des surfaces, a corbure et aire bornees, Ann. Inst. Fourier 38 (1988), 175-203.

[8] A. Futaki, An obstruction to the existence of Einstein Kähler metrics, Inv. Math. Fasc. 73 (1983), no. 3, 437-443. 
[9] Y. Y. Li H. Brezis and I. Shafrir, A sup + inf inequality for some nonliear elliptic equations involving exponential nonlinearities, J. Func. Analysis 115 (1993), 344-358.

[10] J. L. Kazdan and F. W. Warner, Curvature functions for compact 2-manifolds, Ann. of Math 99 (1974), 14-47.

[11] C. Evans Lawrence, Weak convergence for nonlinear partial differential equations, CBMS, AMS, 1990.

[12] M.Troyanov, Prescribing curvature on compact surfaces with conical singularities, Tran. AMS 324 (1991), 793-821.

[13] McOwen R., Point singularities and conformal metrics on Riemann surfaces, Proc. Amer. Math. Soc. 103 (1988), 222-224.

[14] Shioya Takashi, The limit spaces of two dimensional manifolds with uniformly bounded integral curvature, 1996, preprint.

[15] Chen Xiu xiong, Extremal Hermitian metrics with curvature distortion in a surface, Ph.D. thesis, University of Pennsylvania, 1994.

[16] Weak limit of Riemannian metrics in Rieman surfaces and the uniformization theorem, Calculus of Variations 6 (1998), no. 3, 189-226.

$[17]$ , Space of Kähler metrics, submitted to J. D. G., 1998.

[18] S.K. Donaldson , Symmetric spaces, Kahler geometry and Hamiltonian Dynamics, Private communication,1996.

[19] Semmes, S., Complex Monge-Ampere and sympletic manifolds, Amer. J. Math.,114, 495-550, 1992.

[20] T., Mabuchi, Some Sympletic geometry on compact Kähler manifolds I, Osaka, J. Math., 24, 227-252, 1987.

Received September 19, 1997.

[July 27, 1998]

STANFORD UNIVERSITY

STANFORD, CA 94305 